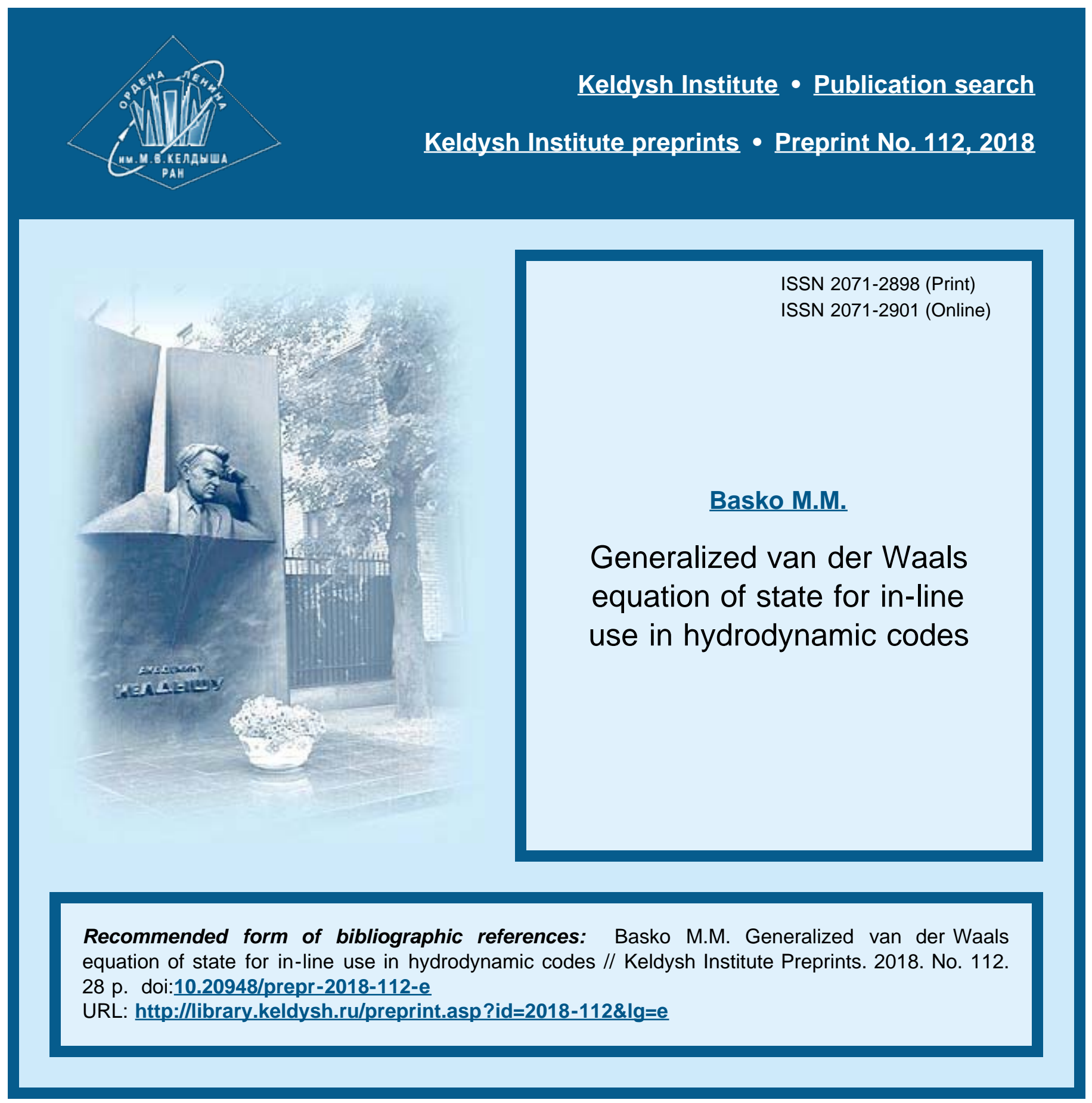




$$
\text { О р д ен а Л ен и на }
$$

ИНСТИТУТ ПРИКЛАДНОЙ МАТЕМАТИКИ

$$
\text { имени М.В. Келдыша }
$$

Р о с с й с кой а кадеми и на ук

\author{
M.M. Basko
}

\title{
Generalized van der Waals equation of state for in-line use in hydrodynamic codes
}




\section{M.M. Basko}

Generalized van der Waals equation of state for in-line use in hydrodynamic codes

Basic physical and mathematical properties of one of the simplest generalizations of the van der Waals equation of state (EOS), where the power exponent $n$ in the attractive term is treated as a free parameter, are investigated. The main focus is on the parameter range around the gas-liquid phase transition, and on the possibility of in-line use of the equilibrium EOS branch (based on the Maxwell construction in the phase coexistence region) in one-dimensional (1D) hydrodynamic simulations. Conditions are elucidated for emergence of such flow structures as a "rarefaction shock" and a "binodal shelf" in rarefaction waves by unloading of compressed matter into vacuum. The quality of numerical modeling of such structures is illustrated with the 1D Lagrangian code DEIRA.

Key words: generalized van der Waals equation of state, hydrodynamic flows with phase transitions.

\section{M.М. Баско}

Обобщенное уравнение состояния ван-дер-Ваальса для использования в режиме in-line в гидродинамических кодах

Представлено исследование основных физических и математических свойств одного из простейших обобщений уравнения состояния ван-дер-Ваальса, в котором дополнительным свободным параметром является показатель степени $n$ в притяжательном члене. Основное внимание уделено области фазового перехода газ-жидкость и возможности прямого (т.е. без промежуточных таблиц) использования равновесной (т.е. полученной с помощью максвелловской конструкции) ветви этого уравнения состояния в одномерных гидродинамических расчетах. Выявлены условия, при которых в волнах разрежения возникают такие особенности течения как "скачок разрежения"и "бинодальная полочка". Качество численного моделирования подобных структур проиллюстрировано с помощью одномерного лагранжевого кода DEIRA.

Ключевые слова: обобщенное уравнение состояния ван-дер-Ваальса, гидродинамические течения с фазовыми переходами. 


\section{Introduction}

In many applications one has to deal with dynamic behavior of liquids and gases that can be well described within the hydrodynamic approach. However, solution of the hydrodynamic equations requires knowledge of the equation of state (EOS), which becomes non-trivial when material undergoes a phase transition. The focus of this work is on the most fundamental and relevant case of the liquid-gas phase transition.

Experiments with ultra-short laser pulses [1, 2] have demonstrated that passage through the liquid-gas phase coexistence region may lead to very specific observational features that are difficult to predict and not easy to explain. Moreover, because of short time scales prevailing in laser experiments, one often confronts a dilemma of choosing between the metastable (MS) and the fully equilibrium (EQ) branches of EOS in the phase coexistence region that is not easy to resolve. Indeed, when the hydrodynamic time scale becomes as short as $\lesssim 1 \mathrm{~ns}$, material in the form of superheated liquid can penetrate deeply into the phase coexistence region along the MS branch of EOS before undergoing a rapid (explosive-like) transition into the EQ state [3].

Thus, it is quite understandable that, whenever one undertakes a theoretical study of some non-trivial aspects of matter hydrodynamics with a liquid-gas phase transition, there arises a need for a two-phase EOS which, on the one hand, (i) would be mathematically as simple as possible - so that it could be implemented into a hydro code directly and used in-line even with the Maxwell construction (producing a numerical noise of only on the order of rounding errors), and, on the other hand, (ii) would be sufficiently realistic - so that, having adjusted its several free parameters, one could reasonably accurately approximate the properties of real materials (like water, liquid metals, etc.).

The simplest analytic EOS, which qualifies for physically adequate description of the liquid-gas phase transition, is the well-known van der Waals equation of state $[4, \S 76]$. As the van der Waals formula only prescribes the dependence of pressure $P(V, T)$ on volume $V$ and temperature $T$, for practical applications it must be augmented by the temperature dependence $c_{V}(T)$ of the heat capacity $c_{V}$ at constant volume, for which usually a constant ideal-gas value is assumed. The main deficiency of the van der Waals EOS is its limited flexibility in description of realistic properties of specific materials. This shortcoming can to a certain extent be overcome by generalizing the van der Waals EOS to a somewhat more complex form, which contains additional free parameters that could be used to fit certain key thermodynamic properties of real materials.

In literature one encounters a number of different analytic equations of state called generalized van der Waals EOS [5, 6, 7, 8, 9]. Here we consider one of them, borrowed from Refs. $[10,11,7]$ and dubbed GWEOS everywhere below, which has 
the same simple functional form as the original van der Waals EOS but includes one more free dimensionless parameter, namely, the power exponent $n>1$ in the attractive-forces correction [see Eqs. (2.3) and (2.4) below]; the original van der Waals EOS is then recovered by setting $n=2$. In this work we present a detailed analysis of certain key properties of GWEOS that are crucial for understanding the principal features of hydrodynamic flows with a liquid-gas phase transition, and illustrate its in-line use within the 1D Lagrangian hydro code DEIRA [12].

\section{Parametrization of GWEOS}

When solving the equations of ideal hydrodynamics, one needs EOS in the form of only one function of two variables, namely, the pressure $P=P(\rho, \epsilon)$ as a function of the mass density $\rho$ and of the mass-specific internal energy $\epsilon$ (or $\epsilon$ as a function of $P$ and $\rho$ ). If, however, the thermal conduction is added, or other processes where the dependence on temperature $T$ is essential, the EOS model must provide two functions of two variables; as those, one usually chooses

$$
P=P(\rho, T) \text { and } \epsilon=\epsilon(\rho, T) .
$$

The above two functions must obey the basic condition

$$
-\rho^{2} \frac{\partial \epsilon}{\partial \rho}=T \frac{\partial P}{\partial T}-P
$$

of thermodynamic consistency.

Because the van der Waals EOS and its numerous modifications have been traditionally discussed in terms of the volume $V$ occupied by $N$ atoms (molecules) as one of the two independent thermodynamic variables [13], we begin by writing our version of the generalized van der Waals EOS (GWEOS) in the form

$$
\begin{aligned}
P & =\frac{T}{V-b}-\frac{a}{V^{n}}, \\
E & =c_{V} T-\frac{a}{n-1} V^{1-n},
\end{aligned}
$$

where $V$ is the volume per one atom (molecule), $E$ is the internal energy per one atom (molecule), and $a>0, b>0, c_{V}>0$, and $n>1$ are constants. The only difference of Eq. (2.3) from the original van der Waals EOS, where $n=2$, is in allowing a free choice of the exponent $n>1$.

Having fixed with Eq. (2.3) the dependence $P(V, T)$, there still remains a functional freedom in temperature dependence of $E(V, T)$ - as is easily verified by invoking the consistency condition (2.2). Here we make the simplest assumption that the thermal component of $E$ is directly proportional to $T$ (a polytropic fluid), 
which leads to Eq. (2.4) where the specific heat $c_{V}$ per atom (molecule) is another free dimensionless (the temperature $T$ is assumed to be in energy units) parameter of the model. For monoatomic substances a natural choice would be $c_{V}=\frac{3}{2}$.

From Eq. (2.3) one readily calculates the parameters of the critical point

$$
V_{c r}=\kappa b, \quad P_{c r}=\frac{a}{\kappa^{n+1} b^{n}}, \quad T_{c r}=\frac{\kappa^{2}-1}{\kappa^{n+1}} \frac{a}{b^{n-1}}=\alpha P_{c r} V_{c r}
$$

where

$$
\kappa=\frac{n+1}{n-1}>1, \quad \alpha=\kappa-\kappa^{-1}=\frac{4 n}{n^{2}-1}>0 .
$$

Expressions (2.5) imply that of the three dimensional quantities $P_{c r}, V_{c r}$ and $T_{c r}$ only two are independent, and that the critical compressibility factor

$$
Z_{c r} \equiv \frac{P_{c r} V_{c r}}{T_{c r}}=\alpha^{-1}=\frac{\kappa}{\kappa^{2}-1}=\frac{n^{2}-1}{4 n}
$$

is a function of the exponent $n$ only. In other words, the extra GWEOS parameter $n$ can be used to fit experimental values of the critical compressibility $Z_{c r}$.

To relate the atomic volume $V$ to the mass density $\rho$, one has to know the mass $M$ of the constituent atoms (molecules)

$$
\rho=\frac{M}{V}, \quad \rho_{c r}=\frac{M}{V_{c r}}
$$

which may be considered as the third (on a par with $P_{c r}$ and $V_{c r}$ ) independent dimensional parameter of the model.

Thus, our GWEOS model has 5 independent free parameters, of which three are dimensional and two dimensionless. Aiming at an EOS in the form (2.1), we choose these 5 free parameters to be

$$
\rho_{c r}, \quad T_{c r}, \quad P_{c r}, \quad c_{V}, \quad n
$$

For any particular substance, the values of $\rho_{c r}, T_{c r}$, and $P_{c r}$ can be simply taken from experiment (when available). Then the effective atomic (molecular) mass $M$ is calculated as

$$
M=\frac{\rho_{c r} T_{c r}}{\alpha P_{c r}}=\frac{n^{2}-1}{4 n} \frac{\rho_{c r} T_{c r}}{P_{c r}} .
$$

If the exponent $n$ is determined from some additional considerations (see section 3.3) rather than from Eq. (2.7), $M$ may differ from the actual atomic (molecular) mass of the considered substance. From the point of view of fluid dynamics this should not be of concern because the hydrodynamic equations are based on the approximation of continuous medium and do not contain $M$. 
Having used the first three parameters from Eq. (2.9) to normalize the main thermodynamic quantities as

$$
\bar{\rho} \equiv v^{-1}=\frac{\rho}{\rho_{c r}}, \quad \theta=\frac{T}{T_{c r}}, \quad p=\frac{P}{P_{c r}}, \quad e=\frac{\rho_{c r}}{P_{c r}} \epsilon,
$$

we can rewrite the two principal GWEOS equations (2.3) and (2.4) in the reduced dimensionless form

$$
\begin{aligned}
p(v, \theta) & =\frac{\alpha \theta}{v-\kappa^{-1}}-\frac{\kappa}{v^{n}}, \\
e(v, \theta) & =c_{V} \alpha \theta-\frac{1}{2} \kappa(\kappa-1) v^{1-n} .
\end{aligned}
$$

This EOS is defined over only a limited range of densities

$$
0<\bar{\rho}<\kappa, \quad \text { i.e. for } v>\kappa^{-1},
$$

while any value of $\theta \geq 0$ is possible. For any $\theta>0$ we have

$$
\lim _{v \rightarrow \kappa^{-1}} p(v, \theta)=+\infty
$$

while the internal energy $e(v, \theta)$ at $v=\kappa^{-1}$ remains finite.

From Eqs. (2.12) and (2.13) one easily derives the expressions

$$
\begin{aligned}
& f(v, \theta)=-\alpha \theta\left[c_{V} \ln \theta+\ln \left(v-\kappa^{-1}\right)\right]-\frac{\kappa(\kappa-1)}{2} v^{1-n} \\
& g(v, \theta)=-\alpha \theta\left[c_{V} \ln \theta+\ln \left(v-\kappa^{-1}\right)-\frac{v}{v-\kappa^{-1}}\right]-\frac{\kappa(\kappa+1)}{2} v^{1-n} \\
& s(v, \theta)=\alpha\left[c_{V}(1+\ln \theta)+\ln \left(v-\kappa^{-1}\right)\right]
\end{aligned}
$$

for the reduced values of the Helmholtz free energy $f$, the Gibbs free energy $g$, and the entropy $s$. Note that the reduced representation preserves all the usual thermodynamic relationships like

$$
d e=\theta d s-p d v, \quad p=-\frac{\partial f}{\partial v}, \quad s=-\frac{\partial f}{\partial \theta}, \quad \ldots
$$

The mass specific values of the above three quantities in conventional units are recovered as

$$
F=\frac{P_{c r}}{\rho_{c r}} f, \quad G=\frac{P_{c r}}{\rho_{c r}} g, \quad S=\frac{P_{c r}}{\rho_{c r} T_{c r}} s .
$$

An important thermodynamic quantity for hydrodynamic simulations is the isentropic speed of sound $c_{s}$. It is calculated from the general expression

$$
c_{s}^{2}=\left(\frac{\partial P}{\partial \rho}\right)_{S}=\left(\frac{\partial P}{\partial \rho}\right)_{T}+\frac{T}{\rho^{2}} \frac{(\partial P / \partial T)_{\rho}^{2}}{(\partial \epsilon / \partial T)_{\rho}} .
$$


Having normalized $c_{s}^{2}$ by the ratio $P_{c r} / \rho_{c r}$,

$$
\bar{c}_{s}^{2}=\frac{\rho_{c r}}{P_{c r}} c_{s}^{2},
$$

we find the square of the dimensionless sound speed to be given by

$$
\bar{c}_{s}^{2}(v, \theta)=\frac{\gamma \alpha \theta v^{2}}{\left(v-\kappa^{-1}\right)^{2}}-\frac{n \kappa}{v^{n-1}},
$$

where

$$
\gamma=1+c_{V}^{-1}
$$

is the adiabatic index in the ideal-gas limit of $v \gg 1$ and/or $\theta \gg 1$. Note that within a certain range of parameters one has $\bar{c}_{s}^{2}<0$, which implies that the hydrodynamic equations cease to be hyperbolic; this region is called the region of non-hyperbolicity.

\section{Properties of the MS-GWEOS}

For any fixed value of $\theta<1$ the pressure isotherms (2.12) exhibit a positive derivative $\partial p(v, \theta) / \partial v>0$ over a certain finite range $v_{s p, l}<v<v_{s p, g}$ (see Fig. 1), which signifies absolute thermodynamic instability [4]. In the parametric $(v, p)$ and $(v, \theta)$ planes, the corresponding region of instability lies under the spinodal - the curve defined by the condition $\partial p(v, \theta) / \partial v=0$. Because such absolutely unstable states would decay on a very short time scale (on the order of 1-10 ps at near-liquid densities), it is reasonable to assume that they would never be reached in hydrodynamic processes. At the same time, the decay time of metastable states above the spinodal (like the timescale of volumetric explosive boiling in a superheated liquid) very rapidly increases even by small deviations from the spinodal $[14,11,3]$, and often very quickly becomes much larger than the relevant hydrodynamic timescale. Such a behavior provides a justification for a simplifying assumption that all the metastable states outside the spinodal, represented by Eqs. (2.12), (2.13), are (at least in principle) accessible via some fast hydrodynamic processes. Accordingly, we denote this metastable branch of GWEOS as MS-GWEOS and assume that, under appropriate circumstances, it can be combined with hydrodynamic equations everywhere above the spinodal. 


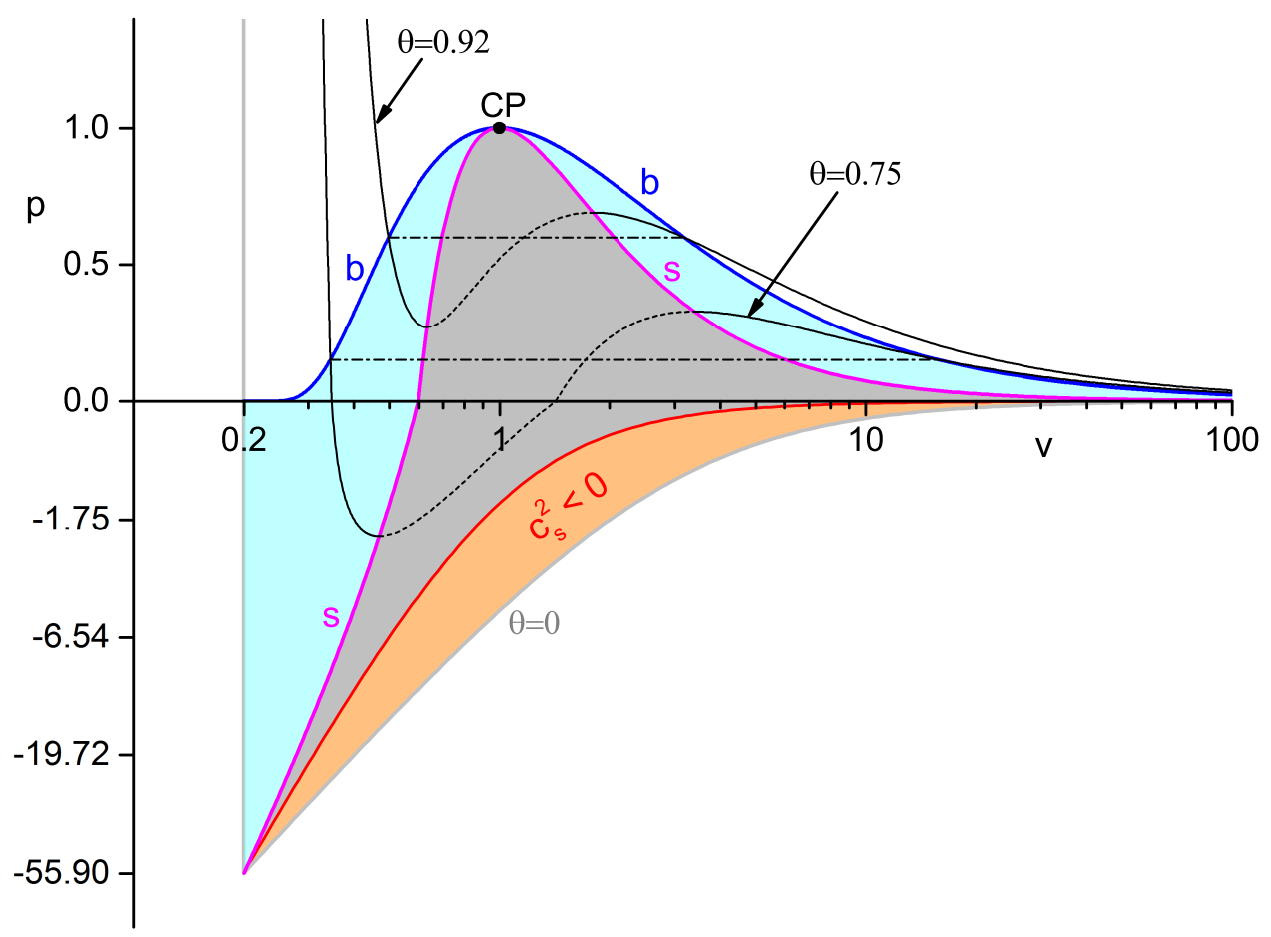

Figure 1: GWEOS with $n=1.5, c_{V}=1.5$ on the thermodynamic $(v, p)$ plane. Metastable states (shaded cyan) lie between the binodal $b$ (blue) and the spinodal $s$ (magenta) curves. The area between the spinodal $s$ and the cold curve $\theta=0$ (shaded grey and grey-orange) is the region of absolute thermodynamic instability that should be considered as inaccessible in hydrodynamic processes with MS EOS. In the region of non-hyperbolicity (shaded grey-orange), where $c_{s}^{2}<0$, the hydrodynamic equations cease to be hyperbolic. Two isotherms $\theta=0.75$ and $\theta=0.92$ are shown as thin black curves in both the metastable (MS, solid curves) and fully equilibrium (EQ, dash-dotted curves) versions of GWEOS. Note that for $p<0$ the vertical axis has a different scale [linear in $\ln (1-p)$ ], which explains the kinks on isotherms and the spinodal by crossing the $p=0$ line.

\subsection{The spinodal}

In the $(v, p)$ and $(v, \theta)$ planes the spinodal is represented by the formulae

$$
\begin{aligned}
& p=p_{s p}(v)=\frac{n+1}{v^{n+1}}\left(v-\frac{1+\kappa^{-1}}{2}\right)=\frac{(n+1) v-n}{v^{n+1}}, \\
& \theta=\theta_{s p}(v)=\frac{1}{v^{n+1}}\left(\frac{v-\kappa^{-1}}{1-\kappa^{-1}}\right)^{2} .
\end{aligned}
$$

In Figs. 1 and 2 these two curves (magenta) are marked with letter $s$. For any value of $0<\theta<1$ Eq. (3.26) has two roots $\kappa^{-1}<v_{s p, l}(\theta)<1$ and $v_{s p, g}(\theta)>1$, belonging, respectively, to the liquid and gaseous branches of the spinodal. Near 


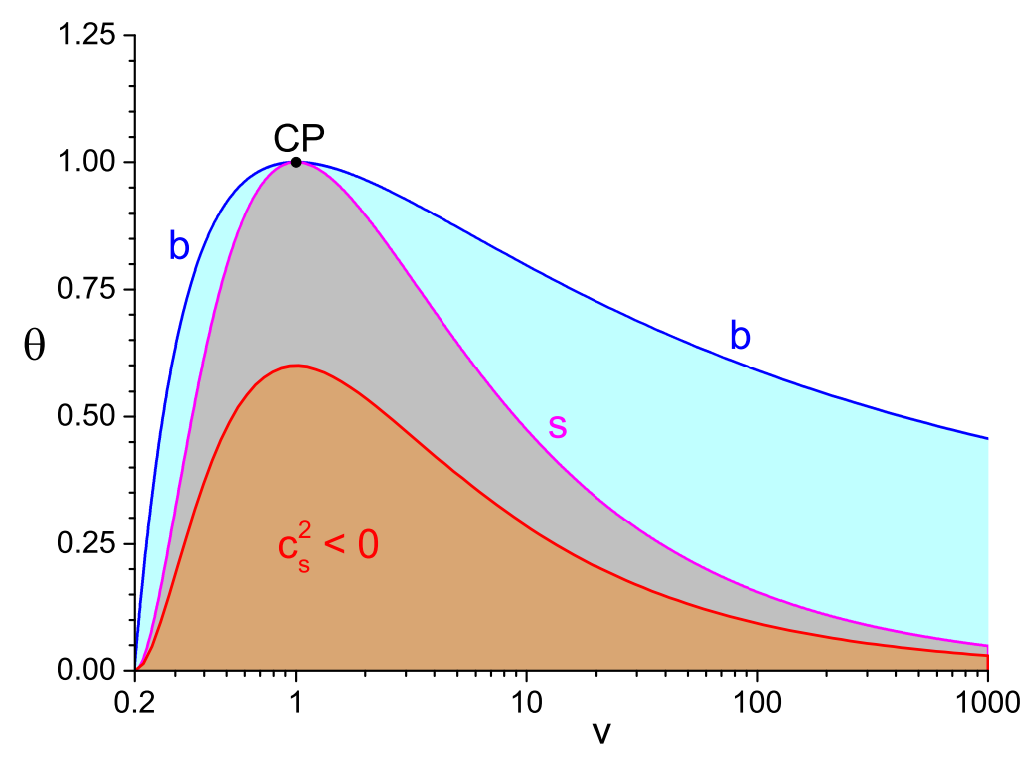

Figure 2: GWEOS with $n=1.5, c_{V}=1.5$ on the thermodynamic $(v, \theta)$ plane. Metastable states (shaded cyan) lie between the binodal $b$ (blue) and the spinodal $s$ (magenta) curves. The area below the spinodal $s$ (shaded grey and greyorange) is the region of absolute thermodynamic instability; the region of nonhyperbolicity, where $c_{s}^{2}<0$, is shaded grey-orange.

the critical point, i.e. at $|v-1| \ll 1$, the first expansion terms are

$$
1-p_{s p}=\frac{n(n+1)}{2}(v-1)^{2}+\ldots, \quad 1-\theta_{s p}=\frac{n^{2}-1}{4}(v-1)^{2}+\ldots
$$

The asymptotic behavior in the limit of $v \rightarrow \infty$ is given by

$$
p_{s p}(v) \rightarrow(n+1) v^{-n}, \quad \theta_{s p}(v) \rightarrow\left(\frac{n+1}{2}\right)^{2} v^{1-n} .
$$

In Fig. 1 one sees that the liquid branch of the spinodal goes all the way down into the negative pressure region and ends at the absolute pressure minimum $p_{s p}\left(\kappa^{-1}\right)=p_{\min } \equiv p\left(\kappa^{-1}, 0\right)=-\kappa^{n+1}$. In the $(v, p)$ plane the spinodal crosses the $p=0$ line at

$$
\begin{aligned}
& v=v_{*}=\frac{1}{2}\left(1+\kappa^{-1}\right)=\frac{n}{n+1}, \\
& \theta=\theta_{*}=\frac{1}{4}\left(\frac{n+1}{n}\right)^{n+1}=\left\{\begin{array}{lll}
0.746496, & n=5, & \kappa=3 / 2, \\
0.84375, & n=2, & \kappa=3, \\
0.89652, & n=3 / 2, & \kappa=5 .
\end{array}\right.
\end{aligned}
$$

The latter means that within the limited temperature and density ranges

$$
0<\theta<\theta_{*}, \quad \kappa_{-1}=\frac{n-1}{n+1}<v<\frac{n}{n+1}=v_{*}
$$


a static sharp (i.e. with a step-like density profile) boundary can exist at $p=0$ between the metastable superheated liquid and vacuum. Despite the fact that such metastable states can be sustained for only a limited time, their lifetime may still be comparable or significantly longer than the duration of ns-long (or shorter) laser pulses, used to heat up liquid targets. In such a case preservation of a sharp stable liquid-vacuum interface may strongly affect the reflectivity of the irradiated sample, with the ensuing impact on the threshold for the onset of the ablative regime of laser illumination. Note that the limiting specific volume $v_{*}$ and temperature $\theta_{*}$ are monotonic functions of the exponent $n$, varying within limits

$$
v_{*}(n)=\left\{\begin{array}{ll}
0.5, & n=1, \\
1, & n=\infty,
\end{array} \quad \theta_{*}(n)= \begin{cases}1, & n=1, \\
\frac{1}{4} \exp (1)=0.67957, & n=\infty .\end{cases}\right.
$$

Combining Eq. (2.23) with Eq. (3.26), one readily verifies that the square of the sound speed on the spinodal,

$$
\bar{c}_{s, s p}^{2}(v)=\frac{\kappa n}{c_{V} v^{n-1}}>0
$$

remains everywhere positive, which justifies the extension of hydrodynamic model with the MS-EOS over the entire region of metastable states. The latter is a consequence of the general thermodynamic inequality

$$
\left(\frac{\partial P}{\partial \rho}\right)_{S}>\left(\frac{\partial P}{\partial \rho}\right)_{T}
$$

implied by Eq. (2.21). The boundary of the non-hyperbolicity region, defined by the condition $c_{s}^{2}=0$, is given by the curves

$$
\begin{aligned}
& p=p_{n h}(v)=\frac{\kappa}{v^{n}}\left[\frac{n c_{V}}{1+c_{V}}\left(1-\frac{1}{\kappa v}\right)-1\right], \\
& \theta=\theta_{n h}(v)=\frac{c_{V}}{1+c_{V}} \frac{1}{v^{n+1}}\left(\frac{v-\kappa^{-1}}{1-\kappa^{-1}}\right)^{2}=\frac{c_{V}}{1+c_{V}} \theta_{s p}(v),
\end{aligned}
$$

which in the $(v, p)$ and $(v, \theta)$ planes lie always below the spinodal curves; see Figs. 1 and 2. Note that for $n<\gamma=1+c_{V}^{-1}$ the curve $p_{n h}(v)$ lies fully in the negative half-plane - as is the case in Fig. 1.

\subsection{Isentropes}

In many types of hydrodynamic flow (like free expansion into vacuum) the fluid entropy is conserved along the trajectories of its mass elements. The corresponding 
equations of isentropes in MS-GWEOS are

$$
\begin{aligned}
& \theta=\theta_{s}(v)=\frac{K_{s}}{\left(v-\kappa^{-1}\right)^{1 / c_{V}}}, \\
& p=p_{s}(v)=\frac{\alpha K_{s}}{\left(v-\kappa^{-1}\right)^{\gamma}}-\frac{\kappa}{v^{n}},
\end{aligned}
$$

where

$$
K_{s}=\exp \left(\alpha^{-1} c_{V}^{-1} s-1\right)
$$

is the entropy constant. More useful for practical applications can be the representation

$$
\begin{aligned}
& \theta_{s}(v)=\theta_{0}\left(\frac{v_{0}-\kappa^{-1}}{v-\kappa^{-1}}\right)^{1 / c_{V}}, \\
& p_{s}(v)=\left(p_{0}+\frac{\kappa}{v_{0}^{n}}\right)\left(\frac{v_{0}-\kappa^{-1}}{v-\kappa^{-1}}\right)^{\gamma}-\frac{\kappa}{v^{n}},
\end{aligned}
$$

where any isentrope is specified by some point on it with coordinates $\left(v_{0}, \theta_{0}\right)$ or $\left(v_{0}, p_{0}\right)$.
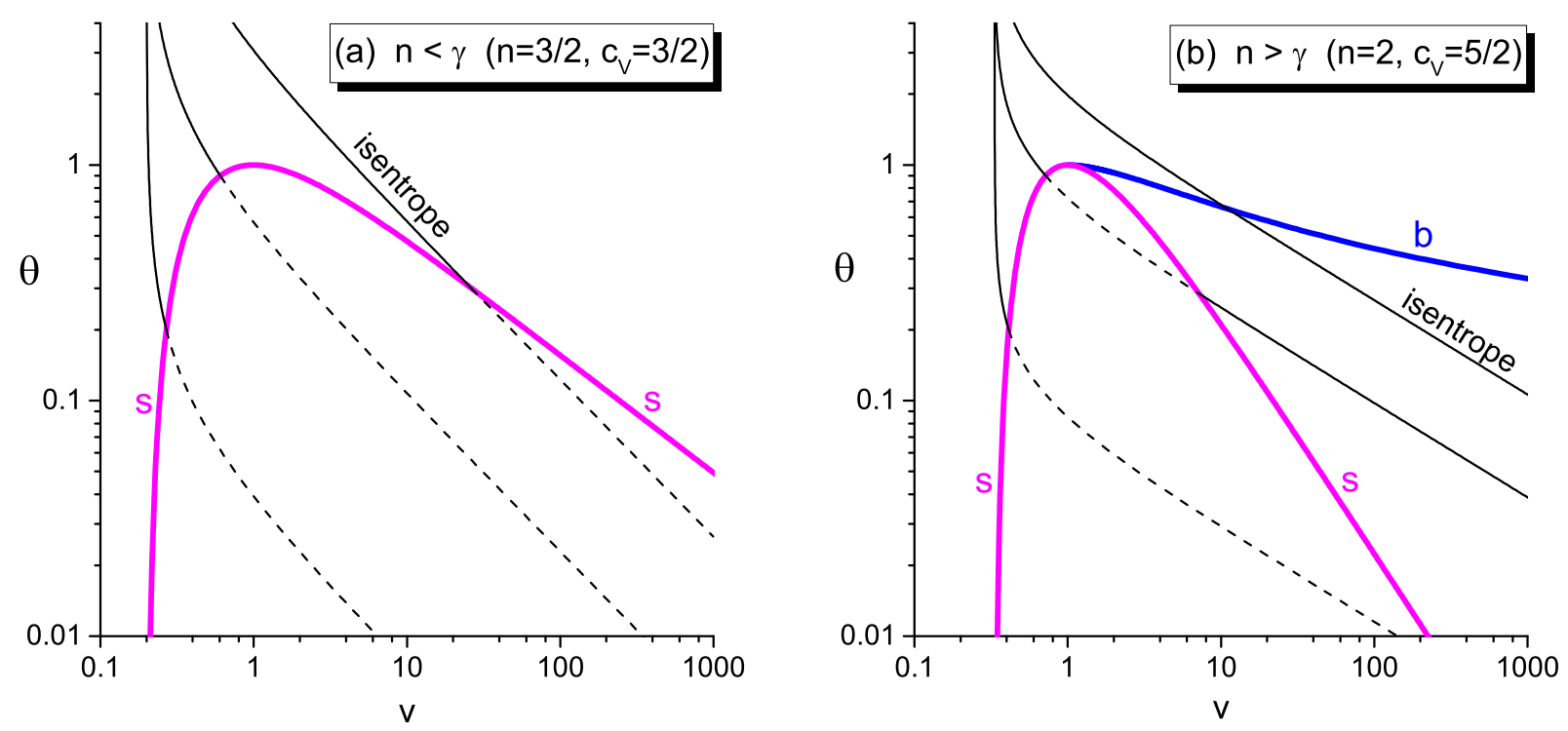

Figure 3: Trajectories of isentropes (thin black curves) relative to the spinodal $s$ (thick magenta curve) for the cases (a) $n<\gamma$ (normal) and (b) $n>\gamma$ (abnormal) on the $(v, \theta)$ plane. Shown in panel (b) is also the vapor branch of the binodal (thick blue curve).

When we juxtapose Eq. (3.38) with the analogous formula (3.25) for the spinodal, we find that, depending on how $\gamma=1+c_{v}^{-1}$ compares with $n$, there are two qualitatively different cases of material behavior by free expansion into vacuum (see Fig. 3): 
(a) for $n<\gamma$ any isentrope (i.e. for any value of $s$ ) earlier or later crosses the spinodal; if this happens at $v<1$, material undergoes volumetric explosive boiling; for sufficiently large entropy values the isentropes cross the spinodal at $v>1$, in which case the expanding material undergoes rapid volumetric condensation;

(b) if $n>\gamma$, sufficiently high isentropes lie everywhere above the spinodal (see Fig. 3b), which implies that the adiabatic expansion of vapor into vacuum may proceed without condensation.

We consider case (a) to be the normal one. It requires $n<5 / 3$ for monoatomic gases where $c_{V}=3 / 2$, and $n<7 / 5$ for two-atomic gases where $c_{V}=5 / 2$. At the same time, it should be noted that none of the expanding isentropes ever comes out from under the binodal, as can be verified by comparing Eq. (3.37) with Eq. (4.72) and is illustrated in Fig. 3b.

\subsection{Cohesive energy and realistic values of $n$}

An important energy characteristic of GWEOS is its cohesive energy, which in reduced variables is given by

$$
e_{c o h}=-\int_{\kappa^{-1}}^{\infty} p_{c}(v) d v=\frac{\kappa^{n}}{n-1}=\frac{1}{2}(\kappa-1) \kappa^{n},
$$

where $p_{c}(v)=p(v, 0)=-\kappa / v^{n}$ is the cold pressure. In the limit of low temperatures $\theta \ll 1$ the cohesive energy becomes equal to the enthalpy of vaporization $h_{l g}$ (see section 4.3 below).

When measured relative to the critical temperature $T_{c r}$, the cohesive energy $E_{\text {coh }}$ per one atom (molecule) varies in a relatively narrow range for different substances. More precisely, if we define the ratio

$$
\Lambda \equiv \frac{E_{c o h}}{T_{c r}}=\frac{M P_{c r}}{\rho_{c r} T_{c r}} e_{c o h}=Z_{c r} e_{c o h}=\frac{(n+1)^{n+1}}{4 n(n-1)^{n}},
$$

then the experimental values of this ratio for a wide range of monoatomic and two-atom molecular substances, for which the parameters of the critical point have been reliably measured, vary in the range $\Lambda \approx 4.0-5.3$ [15]. These values of $\Lambda$ can be reproduced in GWEOS by setting $n=1.4-1.65$. The best-fit values of $n$, quoted in Ref. [7] for alkali metals, nicely fall in this range. Note that, when the goal is to improve the adequacy of hydrodynamic modeling, determination of $n$ from the experimental values of $\Lambda$ appears more appropriate than from the values of $Z_{c r}$ because a more adequate target reaction to the external energy deposition 
can be expected in this case - although both criteria usually yield close results. In general, one can adopt $n=1.5$ as a universal value that can to a reasonable accuracy describe thermodynamic properties of most monoatomic substances (for which one would assume $c_{V}=3 / 2$ ) near the region of liquid-gas phase transition.

\subsection{Negative non-linearity and rarefaction shocks}

An important role in hydrodynamics belongs to the so called fundamental gasdynamic derivative [16]

$$
\Gamma \equiv \frac{v}{2}\left(\frac{\partial p}{\partial v}\right)_{s}^{2}\left(\frac{\partial^{2} v}{\partial p^{2}}\right)_{s}=-\frac{v}{2} \frac{d^{2} p_{s} / d v^{2}}{d p_{s} / d v}=1+\frac{\bar{\rho}}{\bar{c}_{s}}\left(\frac{\partial \bar{c}_{s}}{\partial \bar{\rho}}\right)_{s},
$$

where $p_{s}(v)$ is the equation of isentrope. The sign of this derivative determines admissible types of the shock-wave solutions in ideal hydrodynamics. If $\Gamma>$ 0 everywhere, we have the normal case of positive non-linearity, where only compression shocks can exist. If $\Gamma<0$ everywhere, we deal with an abnormal case of negative non-linearity (NNL), where only rarefaction shocks can exist. For a perfect polytropic gas with $p_{s}(v) \propto v^{-\gamma}$ we have $\Gamma=\frac{1}{2}(\gamma+1)$.

It has been known [17] that the original van der Waals EOS exhibits a limited NNL region near the critical point above the binodal. For the GWEOS considered here we calculate

$$
\Gamma(v, \theta)=\frac{1}{2} \frac{\gamma(\gamma+1)\left(1-\kappa^{-2}\right) \theta v^{n+2}\left(v-\kappa^{-1}\right)^{-3}-n(n+1)}{\gamma\left(1-\kappa^{-2}\right) \theta v^{n+1}\left(v-\kappa^{-1}\right)^{-2}-n} .
$$

To obtain $\Gamma(v, p)$ from $\Gamma(v, \theta)$, one has to make a substitution

$$
\left(1-\kappa^{-2}\right) \theta=\left(p \kappa^{-1}+v^{-n}\right)\left(v-\kappa^{-1}\right) .
$$

Below we use also the quantity $\Gamma_{\infty}(v, \theta)$, obtained by setting $\gamma=1$ in Eq. (3.45); it corresponds to the limiting case of $c_{V}=\infty$.

Because we treat all the metastable states between the binodal and the spinodal on equal basis with the absolutely stable ones, we are interested in a broader region of negative non-linearity that would lie above the spinodal curve - in contrast to the one above the binodal analyzed by previous authors $[17,18,19]$. The boundary $\Gamma=0$ of the NNL region in GWEOS is obtained by setting the numerator in Eq. (3.45) equal to zero; the denominator is everywhere positive above and on the spinodal because it becomes zero only along the nonhyberbolicity boundary $c_{s}^{2}=0$, which lies below the spinodal (see section 3.1 ). 


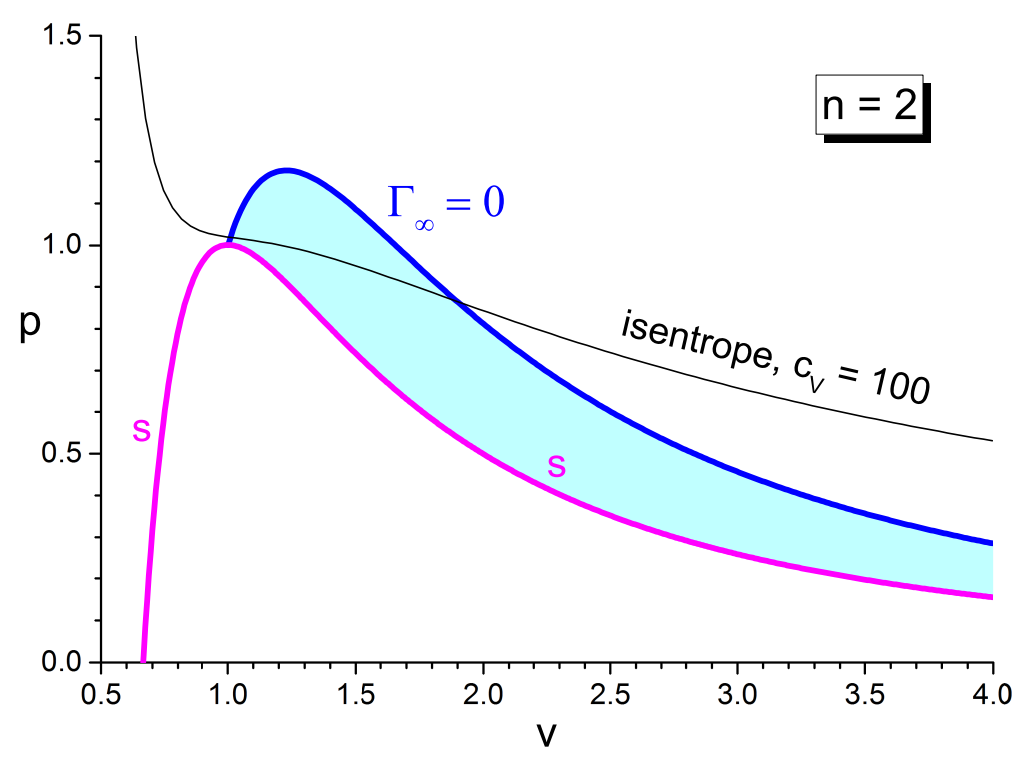

Figure 4: Region of negative non-linearity (shaded cyan) between the spinodal $s$ (thick magenta) and the limiting NNL boundary $\Gamma_{\infty}=0$ (thick blue) on the $(v, p)$ plane for the van der Waals EOS $(n=2)$. Shown also is an isentrope, calculated for $c_{V}=100$ and lying slightly above the critical point. One clearly sees an abnormal convex segment of this isentrope inside the NNL region.

From Eqs. (3.45) and (3.46) we obtain the following equations for this boundary

$$
\begin{aligned}
\theta=\theta_{n n l}(v) & =\frac{2}{\gamma(\gamma+1)}\left(\frac{v-\kappa^{-1}}{1-\kappa^{-1}}\right)^{3} \frac{1}{v^{n+2}}= \\
& =\frac{2}{\gamma(\gamma+1)}\left(1+\frac{1-\bar{\rho}}{\kappa-1}\right) \theta_{s p}(v), \\
p=p_{n n l}(v) & =\frac{\kappa}{v^{n}}\left[\frac{n(n+1)}{\gamma(\gamma+1)}\left(1-\frac{1}{\kappa v}\right)^{2}-1\right] .
\end{aligned}
$$

On the $(v, \theta)$ plane the NNL region is defined by the condition

$$
\theta_{s p}(v)<\theta<\theta_{n n l}(v)
$$

Because $\gamma>1$, Eq. (3.47) implies that the whole of the NNL region lies at $\bar{\rho}=v^{-1}<1$, and it first appears at $\bar{\rho}=0$ once the condition

$$
\gamma<\gamma_{n n l}=\frac{\sqrt{4 n+5}-1}{2}
$$

or, equivalently,

$$
c_{V}>c_{V, n n l}=\frac{\sqrt{4 n+5}+3}{2(n-1)}= \begin{cases}3.303, & n=2, \\ 6.317, & n=3 / 2,\end{cases}
$$


is fulfilled. The maximum possible extension of the NNL region in the $(v, p)$ plane in the limit of $c_{V} \rightarrow \infty$ for the van der Waals EOS is shown in Fig. 4 as the area between the spinodal $s$ and the curve $\Gamma_{\infty}=0$, which is calculated by setting $\gamma=1, n=2$ in Eq. (3.48). Note that in the case of $\gamma>n$, designated as the normal one in section 3.2, the NNL region does not exist because $\gamma_{n n l}<n$. One also notices that the smaller the $n$ value, the more narrow is the NNL region.

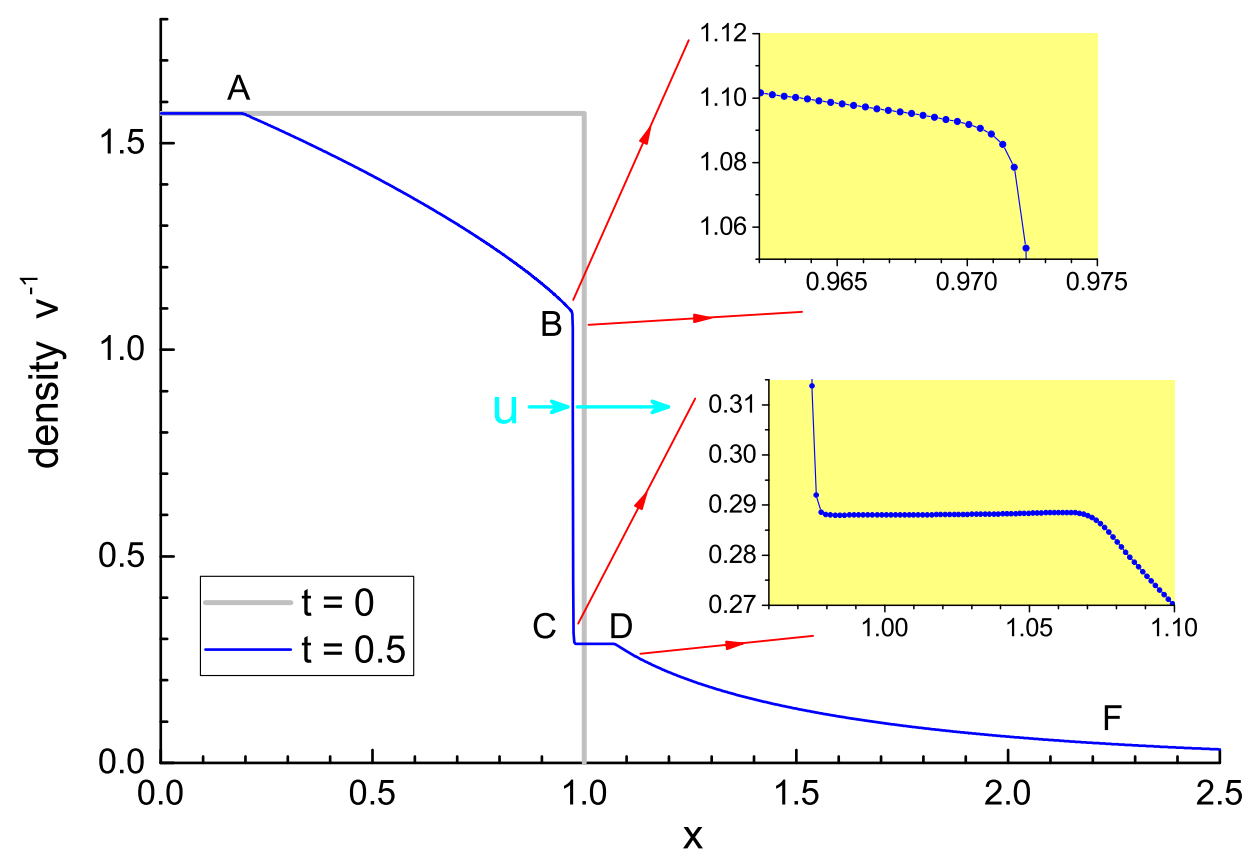

Figure 5: Rarefaction shock calculated with the DEIRA hydrodynamic code for the van der Waals EOS $\left(n=2, c_{V}=100\right)$. The initial state at point $A$ lies on the isentrope of Fig. 4 at $p=p_{0}=1.505$. The inserts with yellow background, where dots represent individual grid cells, illustrate the numerical accuracy near the kink point $B$ and the supersonic plateau $C D$. The two horizontal arrows (cyan) indicate the jump of material velocity $u$ across the shock front $B C$.

To illustrate what happens in the NNL region, Fig. 4 shows also an isentrope, calculated for $n=2$ and $c_{V}=100$, which starts at a point $\left(v_{0}, p_{0}\right)=(0.636,1.505)$ and passes through the NNL region slightly above the critical point. Because it has a convex segment inside the NNL region, unloading into vacuum along this isentrope cannot proceed as a smooth rarefaction wave. As is explained in $\S 20$ of chapter XI in Ref. [20], any initially smooth unloading profile along such an isentrope would inevitably develop a discontinuity - a rarefaction shock, encompassing the convex segment on the $(v, p)$ plane. When the expanding material passes through the rarefaction shock front, its entropy increases, and the resulting unloading wave is no longer isentropic. 
Figure 5 displays the numerical solution for the unloading profile at $t=0.5$, starting at $t=0$ from the step function with the initial state on the isentrope of Fig. 4 at $v=v_{0}=0.636, p=p_{0}=1.505$. This solution was obtained with the one-dimensional (1D) Lagrangian code DEIRA [12] on a grid with 2000 cells over the interval $0<x<1$. From the evolutionary and stability considerations one would expect that the exact solution (which is self-similar) must exhibit a so called double-sonic rarefaction shock [18], where the upstream and downstream Mach numbers with respect to the shock front are equal to one. The numerical solution in Fig. 5 does indeed demonstrate the upstream Mach number $\mathcal{M}_{B}=1.00$ at the entrance point $B$. However, the downstream value $\mathcal{M}_{C}=1.16$ turns out to be noticeably larger than unity, which indicates a supersonic exit velocity at point $C$. As a result, there develops a plateau $C D$, whose width grows linearly with time because the fluid passes through the kink point $D$ with exactly the local sound velocity; obviously, in a double-sonic shock the points $C$ and $D$ should coincide.

The appearance of a supersonic plateau $C D$ in our illustrative example must be attributed to inaccuracies associated with the specific finite-difference scheme of the DEIRA code. Numerical tests demonstrate that the position and width of the supersonic plateau are not sensitive to the number of grid cells and other parameters of the numerical scheme, whereas the EOS is calculated in-line with the accuracy of 15 digits. It can probably be explained by the stepwise initial condition, for which the formation of the entire self-similar expansion profile always begins with a single mesh cell at the boundary with vacuum. Finitedifference errors manifest themselves also in smoothed corners of the density profile at points $B, C$ and $D$, where the exact profile $\bar{\rho}(x)$ has a discontinuous first derivative. The numerical solution with a supersonic plateau behind the rarefaction shock is mechanically stable because the influence of the boundary condition from the vacuum side cannot reach beyond the "sonic" point $D$. Note that in all other respects the numerical solution is quite accurate: apart from the kink points, the relative errors in the density (pressure) profiles are confined to $\lesssim 10^{-3}$; the entropy along the smooth segments $A B$ and $D F$ is conserved with 4-5 digits. At the same time, the entropy jump in the exact double-sonic rarefaction shock is so small $\left(\lesssim 10^{-4}\right)[18]$ that it cannot be discerned in the present numerical solution.

\section{Fully equilibrium EQ-GWEOS}

The easiest and most straightforward way to do hydrodynamic simulations with liquid-gas phase transitions is by using the fully equilibrium (EQ) EOS, obtained by applying the Maxwell rule inside the phase coexistence region. 


\subsection{The binodal}

For any given value of temperature $\theta<1$ the liquid-gas phase transition occurs within a certain interval of specific volumes

$$
v_{l}(\theta)<v<v_{g}(\theta), \quad \theta<1 .
$$

The curves $v=v_{l}(\theta)<1$ and $v=v_{g}(\theta)>1$ represent, respectively, the liquid and the vapor branches of the binodal, shown in Fig. 2 for $n=1.5$. On the $(v, p)$ plane in Fig. 1 the liquid and the vapor binodal branches are given by parametric curves $\left\{p=p_{\text {sat }}(\theta), v=v_{l}(\theta)\right\}$ and $\left\{p=p_{\text {sat }}(\theta), v=v_{g}(\theta)\right\}$, where

$$
p_{\text {sat }}(\theta)=p\left(v_{l}(\theta), \theta\right)=p\left(v_{g}(\theta), \theta\right),
$$

the temperature $0<\theta<1$ serves as the curve parameter, and $p(v, \theta)$ is given by Eq. (2.12).

The EQ-GWEOS differs from the MS-GWEOS, given by Eqs. (2.12), (2.13), (2.16)-(2.18), only within the phase coexistence region (4.52), i.e. under the binodal curve. For any given temperature $\theta<1$, the densities of the boiling liquid $\bar{\rho}_{l}=v_{l}^{-1}$ and of the saturated vapor $\bar{\rho}_{g}=v_{g}^{-1}$ are found by solving the system of two equations

$$
\begin{aligned}
& \theta\left[\ln \frac{\kappa / \bar{\rho}_{g}-1}{\kappa / \bar{\rho}_{l}-1}+\frac{\kappa}{\kappa-\bar{\rho}_{l}}-\frac{\kappa}{\kappa-\bar{\rho}_{g}}\right]=\frac{\kappa^{2}}{2(\kappa-1)}\left(\bar{\rho}_{l}^{n-1}-\bar{\rho}_{g}^{n-1}\right), \\
& \theta=\frac{1}{\kappa^{2}-1} \frac{\bar{\rho}_{l}^{n}-\bar{\rho}_{g}^{n}}{\bar{\rho}_{l}-\bar{\rho}_{g}}\left(\kappa-\bar{\rho}_{l}\right)\left(\kappa-\bar{\rho}_{g}\right) .
\end{aligned}
$$

These equations express the conditions that equilibrium coexistence of two different phases requires that their Gibbs free energies $g(v, \theta)$ and their pressures $p(v, \theta)$ be equal [4]. Having substituted Eq. (4.55) into Eq. (4.54), we obtain an equivalent form of (4.54)

$$
\frac{\left(\kappa-\bar{\rho}_{l}\right)\left(\kappa-\bar{\rho}_{g}\right)}{\kappa\left(\bar{\rho}_{l}-\bar{\rho}_{g}\right)} \ln \frac{\kappa / \bar{\rho}_{g}-1}{\kappa / \bar{\rho}_{l}-1}+1=\frac{\kappa(\kappa+1)}{2} \frac{\bar{\rho}_{l}^{n-1}-\bar{\rho}_{g}^{n-1}}{\bar{\rho}_{l}^{n}-\bar{\rho}_{g}^{n}},
$$

which does not contain $\theta$.

Given the value of $\theta<1$, the numerical solution of Eqs. (4.54), (4.55) is achieved in a double iteration loop to the accuracy of about 15 digits. From the numerical point of view, it is convenient to choose $\bar{\rho}_{l}$ and $x=\ln v_{g}=-\ln \bar{\rho}_{g}$ as the two unknown variables (because in practice the values of $x \gtrsim 10^{3}-10^{4}$ may be encountered), for which the roots of Eqs. (4.54) and (4.55) must be found inside the intervals

$$
\bar{\rho}_{s p, l}(\theta)<\bar{\rho}_{l}<\kappa, \quad-\ln \bar{\rho}_{s p, g}(\theta)<x<\frac{\kappa^{n+1}}{2(\kappa+1)} \frac{1}{\theta}+\ln (\theta / \kappa) .
$$


Here $v_{s p, l}(\theta)=1 / \bar{\rho}_{s p, l}(\theta)<1$ and $v_{s p, g}(\theta)=1 / \bar{\rho}_{s p, g}(\theta)>1$ are, respectively, the liquid and the vapor roots of the spinodal equation (3.26); the upper bound on $x$ in Eq. (4.57) is established on the basis of the asymptotic formula (4.72). Once a certain value of $x=\ln v_{g}$ is chosen, Eq. (4.55) may be considered as defining the function $\bar{\rho}_{l}(x)$; calculation of this function with 15 digits requires on average about 30 iterations (in the particular implementation of the EQ-GWEOS in the DEIRA code). Then, with the function $\bar{\rho}_{l}(x)$ known, one needs on average about 20 iterations to solve the equation (4.54) for $x$. Thus, the computational cost of the in-line implementation of the EQ-GWEOS is roughly equal to 600 calculations of the $n$-th power of a real number. As a result, computation of the in-line EQ-GWEOS becomes the main consumer of the CPU time in 1D hydrodynamic simulations, with the final slowdown relative to the MS-GWEOS option by about a factor 100. For 1D simulations this is still affordable.

\subsection{Thermodynamic functions of the EQ-GWEOS}

Having calculated the coordinates of the binodal $v_{l}(\theta)$ and $v_{g}(\theta)$ from Eqs. (4.54) and (4.55), one readily obtains all the thermodynamic functions of the EQGWEOS in the phase coexistence region $v_{l}(\theta)<v<v_{g}(\theta)$ by recalling that, for any fixed $\theta$, the pressure $p=p_{E Q}(v, \theta)$ is independent of $v$,

$$
p_{E Q}(v, \theta)=p_{\text {sat }}(\theta)=p\left(v_{l}(\theta), \theta\right)=p\left(v_{g}(\theta), \theta\right),
$$

while the entropy $s=s_{E Q}(v, \theta)$ and all the thermodynamic potentials $e=$ $e_{E Q}(v, \theta), f=f_{E Q}(v, \theta), g=g_{E Q}(v, \theta)$ are linear functions of $v$,

$$
\begin{aligned}
& s_{E Q}(v, \theta)=\alpha\left[c_{V}(1+\ln \theta)+\nu_{l} \ln \left(v_{l}-\kappa^{-1}\right)+\nu_{g} \ln \left(v_{g}-\kappa^{-1}\right)\right], \\
& e_{E Q}(v, \theta)=c_{V} \alpha \theta-\frac{1}{2} \kappa(\kappa-1)\left(\nu_{l} \bar{\rho}_{l}^{n-1}+\nu_{g} \bar{\rho}_{g}^{n-1}\right),
\end{aligned}
$$

where

$$
\nu_{l}(v, \theta)=\frac{v_{g}(\theta)-v}{v_{g}(\theta)-v_{l}(\theta)}, \quad \nu_{g}(v, \theta)=1-\nu_{l}(v, \theta)=\frac{v-v_{l}(\theta)}{v_{g}(\theta)-v_{l}(\theta)},
$$

are, respectively, the mass fractions of the liquid and vapor phases in the considered two-phase state with $v_{l}<v<v_{g}$. The Helmholtz free energy $f_{E Q}=e_{E Q}-\theta s_{E Q}$ and the Gibbs free energy $g_{E Q}=f_{E Q}+v p_{E Q}$ are readily recovered from Eqs. (4.59) and (4.60).

Beside the values of the main thermodynamic functions $p_{E Q}$ and $e_{E Q}$, one needs also their first derivatives. For the pressure derivatives the situation is relatively simple,

$$
\left(\frac{\partial p_{E Q}}{\partial v}\right)_{\theta}=0, \quad\left(\frac{\partial p_{E Q}}{\partial \theta}\right)_{v}=\frac{d p_{s a t}}{d \theta}=\frac{s_{g}-s_{l}}{v_{g}-v_{l}}=\frac{\alpha}{v_{g}-v_{l}} \ln \frac{v_{g}-\kappa^{-1}}{v_{l}-\kappa^{-1}},
$$


where the second formula is the well-known Clausius-Clapeyron relation, with

$$
h_{l g}(\theta)=\theta\left(s_{g}-s_{l}\right)=\alpha \theta \ln \frac{v_{g}-\kappa^{-1}}{v_{l}-\kappa^{-1}}
$$

being the enthalpy of vaporization. Then, from the principal thermodynamic identity (2.2) one calculates

$$
\left(\frac{\partial e_{E Q}}{\partial v}\right)_{\theta}=\theta\left(\frac{\partial p_{E Q}}{\partial \theta}\right)_{v}-p_{E Q}
$$

and there remains only one non-trivial first derivative, namely, the heat capacity by constant volume

$$
\begin{aligned}
\left(\frac{\partial e_{E Q}}{\partial \theta}\right)_{v} & =\alpha c_{V}+\frac{1}{2} \kappa(\kappa-1)\left(\Sigma_{l} \frac{1}{v_{l}} \frac{d v_{l}}{d \theta}+\Sigma_{g} \frac{1}{v_{g}} \frac{d v_{g}}{d \theta}\right) \\
\Sigma_{l} & =-v_{l} \frac{\partial}{\partial v_{l}}\left(\nu_{l} \bar{\rho}_{l}^{n-1}+\nu_{g} \bar{\rho}_{g}^{n-1}\right)= \\
& =\nu_{l}\left(n \bar{\rho}_{l}^{n-1}-\bar{\rho}_{g}^{n-1}+\frac{\bar{\rho}_{g}^{n-1}-\bar{\rho}_{l}^{n-1}}{1-\bar{\rho}_{g} / \bar{\rho}_{l}}\right), \\
\Sigma_{g} & =-v_{g} \frac{\partial}{\partial v_{g}}\left(\nu_{l} \bar{\rho}_{l}^{n-1}+\nu_{g} \bar{\rho}_{g}^{n-1}\right)= \\
& =\nu_{g}\left[(n-1) \bar{\rho}_{g}^{n-1}+\frac{\bar{\rho}_{g}^{n-1}-\bar{\rho}_{l}^{n-1}}{1-\bar{\rho}_{g} / \bar{\rho}_{l}}\right], \\
\frac{1}{v_{l}} \frac{d v_{l}}{d \theta} & =\left(\frac{d p_{s a t}}{d \theta}-\frac{\alpha \bar{\rho}_{l}}{1-\bar{\rho}_{l} / \kappa}\right)\left[\kappa n \bar{\rho}_{l}^{n}-\frac{\alpha \theta \bar{\rho}_{l}}{\left(1-\bar{\rho}_{l} / \kappa\right)^{2}}\right]^{-1} \\
\frac{1}{v_{g}} \frac{d v_{g}}{d \theta} & =\left(\frac{d p_{s a t}}{d \theta}-\frac{\alpha \bar{\rho}_{g}}{1-\bar{\rho}_{g} / \kappa}\right)\left[\kappa n \bar{\rho}_{g}^{n}-\frac{\alpha \theta \bar{\rho}_{g}}{\left(1-\bar{\rho}_{g} / \kappa\right)^{2}}\right]^{-1} .
\end{aligned}
$$

Having calculated $\left(\partial e_{E Q} / \partial \theta\right)_{v}$, one obtains the square of the sound velocity from

$$
\bar{c}_{s, E Q}^{2}=\theta\left(v \frac{d p_{s a t}}{d \theta}\right)^{2}\left(\frac{\partial e_{E Q}}{\partial \theta}\right)_{v}^{-1}
$$

\subsection{Asymptotic behavior at $\theta<<1$}

At low temperatures $\theta \ll 1$ the density $\bar{\rho}_{g}(\theta)$ of the saturated vapor approaches zero faster than any finite power of $\theta$, namely, as $\exp (-a / \theta)$, where $a$ is a 
constant (the Arrhenius law). Taking this into account, one readily calculates from Eq. (4.55) the first three expansion terms of $\bar{\rho}_{l}(\theta)$ in powers of $\theta$

$$
\bar{\rho}_{l}(\theta)=\kappa-\frac{\kappa^{2}-1}{\kappa^{n}} \theta\left[1+\frac{2(\kappa+1)}{\kappa^{n+1}} \theta\right]+O\left(\theta^{3}\right) .
$$

Then, having substituted Eq. (4.71) into Eq. (4.56), one obtains the asymptotic formulae

$$
\begin{aligned}
v_{g}(\theta) & \rightarrow \frac{\kappa^{2}-1}{\kappa^{n+2}} \theta \exp \left[\frac{\kappa^{n+1}}{2(\kappa+1)} \frac{1}{\theta}\right], \\
p_{\text {sat }}(\theta) & \rightarrow \kappa^{n+1} \exp \left[-\frac{\kappa^{n+1}}{2(\kappa+1)} \frac{1}{\theta}\right],
\end{aligned}
$$

for the specific volume and pressure of the saturated vapor along the gaseous branch of the binodal in the limit of $\theta \rightarrow 0$. Note that the above expressions differ by a pre-exponential factor of $\exp (1)$ from the analogous asymptotics calculated in Ref. [21] for the case of $n=2$.

Because

$$
v_{l}(\theta)-\kappa^{-1}=\frac{\kappa^{2}-1}{\kappa^{n+2}} \theta+O\left(\theta^{2}\right),
$$

Eq. (4.73) implies that the liquid branch of the binodal on the $(v, p)$ plane touches the horizontal $v$-axis at $v=\kappa^{-1}$ with an infinite-order tangency, i.e. with $d^{m} p / d v^{m}=0$ for all $m=1,2, \ldots$, - which is vividly illustrated by the $b$-curve in Fig. 1. Also, one readily verifies that in the limit of $\theta \rightarrow 0$ the enthalpy of vaporization

$$
h_{l g}(\theta)=\alpha \theta \ln \frac{v_{g}-\kappa^{-1}}{v_{l}-\kappa^{-1}} \rightarrow \frac{1}{2}(\kappa-1) \kappa^{n}=e_{c o h}
$$

approaches the cohesive energy $e_{\text {coh }}$, defined in Eq. (3.42).

When solving Eqs. (4.54) and (4.55) for the values of $v_{l}(\theta)$ and $v_{g}(\theta)$, it is helpful to have a simple estimate for the upper bound on $v_{g}(\theta)$. Starting from the asymptotic formula (4.72), one can suggest an expression

$$
v_{g, \text { sup }}(\kappa, \theta)=\frac{\theta}{\kappa} \exp \left[\frac{\kappa^{n+1}}{2(\kappa+1)} \frac{1}{\theta}\right]>\frac{\kappa^{2}-1}{\kappa^{n+2}} \theta \exp \left[\frac{\kappa^{n+1}}{2(\kappa+1)} \frac{1}{\theta}\right] .
$$

On the one hand, inequality in Eq. (4.76) guarantees that $v_{g, \text { sup }}(\kappa, \theta)>v_{g}(\theta)$ in the limit of $\theta \ll 1$. On the other hand, one easily verifies that $v_{g, s u p}(\kappa, 1)>6$ for any $\kappa>1$, which in practice implies $v_{g, s u p}(\kappa, \theta)>v_{g}(\theta)$ for all $\theta<1$ and $\kappa>1$. 


\subsection{Expansion near the critical point}

We begin by calculating the curvature $2 \beta$ of the binodal at the critical point, having assumed that in the vicinity of the critical point, where $1-\theta \ll 1$ and $|v-1| \ll 1$, the equation of the binodal on the $(v, \theta)$ plane can be written as

$$
t \equiv 1-\theta=\beta(v-1)^{2}+O\left[(v-1)^{3}\right] .
$$

Then, having substituted

$$
\bar{\rho}_{l}=1+\sqrt{\frac{t}{\beta}}, \quad \bar{\rho}_{g}=1-\sqrt{\frac{t}{\beta}},
$$

into Eq. (4.55), we get

$$
\beta=\frac{n^{2}-1}{12}=\frac{\kappa}{3(\kappa-1)^{2}} .
$$

The same value of $\beta$ is also calculated when the expansion (4.78) is substituted into Eq. (4.54), which assures the consistency of the procedure.

As $\theta \rightarrow 1-0$, the enthalpy of vaporization $h_{l g}$, given by Eq. (4.63), approaches zero as

$$
h_{l g}(\theta) \rightarrow \frac{2 \alpha}{1-\kappa^{-1}} \sqrt{\frac{t}{\beta}}=2\left(\kappa^{2}-1\right) \sqrt{\frac{3}{\kappa}(1-\theta)} .
$$

The latter implies that the pressure derivative $(\partial p / \partial \theta)_{v}$ is continuous at the critical point because

$$
\lim _{\theta \rightarrow 1-0}\left(\frac{\partial p_{E Q}}{\partial \theta}\right)_{v}=\lim _{\theta \rightarrow 1} \frac{h_{l g}(\theta)}{\theta\left(v_{g}-v_{l}\right)}=\kappa+1=\lim _{\theta \rightarrow 1+0}\left(\frac{\partial p}{\partial \theta}\right)_{v=1} .
$$

In view of the basic identity $(2.2)$, continuity of $(\partial p / \partial \theta)_{v}$ ensures also the continuity of the derivative $(\partial e / \partial v)_{\theta}$, whereas the heat capacity $(\partial e / \partial \theta)_{v}$ turns out to be discontinuous at the critical point. Indeed, if one sets $v=1$ and substitutes into Eq. (4.60)

$$
v_{l}=1-x_{l}, \quad v_{g}=1+x_{g},
$$

where $0<x_{l} \ll 1$ and $0<x_{g} \ll 1$, one calculates the expansion series

$$
\begin{aligned}
e_{E Q}(1, \theta)= & \alpha c_{V}-\frac{\kappa}{n-1}-\alpha c_{V} t-\frac{\kappa n}{2} x_{l} x_{g}\left[1+\frac{n+1}{3}\left(x_{l}-x_{g}\right)+\right. \\
& \left.+\frac{(n+1)(n+2)}{3 \cdot 4}\left(x_{l}^{2}-x_{l} x_{g}+x_{g}^{2}\right)+\ldots\right] .
\end{aligned}
$$


With $x_{l}=x_{g}=\sqrt{t / \beta}$ one obtains

$$
\lim _{\theta \rightarrow 1-0}\left(\frac{\partial e_{E Q}(1, \theta)}{\partial \theta}\right)_{v}=\alpha c_{V}+\frac{\kappa n}{2 \beta}=\alpha\left(c_{V}+\frac{3}{2} \kappa\right)
$$

whereas

$$
\lim _{\theta \rightarrow 1+0}\left(\frac{\partial e(1, \theta)}{\partial \theta}\right)_{v}=\alpha c_{V}
$$

As a direct consequence from Eq. (4.70), discontinuous also is the adiabatic sound speed

$$
\lim _{v=1, \theta \rightarrow 1+0} \bar{c}_{s}^{2}=\frac{\kappa n}{c_{V}}, \quad \lim _{v=1, \theta \rightarrow 1-0} \bar{c}_{s, E Q}^{2}=\frac{\kappa n}{c_{V}+\frac{3}{2} \kappa} .
$$

\section{Illustrative example: unloading of a planar slab into vacuum}

To illustrate the implementation of the EQ-GWEOS in the in-line mode, one of the simplest ideal-hydrodynamics 1D problems, namely, the unloading of an initially uniform planar slab into vacuum, was simulated with the DEIRA code for the GWEOS parameters $n=1.5, c_{V}=1.5$. The corresponding unloading isentrope $A B C$ is shown in Fig. 6. Initially, the motionless material is confined to a layer $-1<x<+1$ in a uniform state with $\bar{\rho}=\bar{\rho}_{0}=2.92194, \theta=\theta_{0}=1.332594$, $p=p_{0}=19.99656$ (point $A$ in Fig. 6). The unloading wave into vacuum starts at $t=0$ from both ends of the slab, where the zero boundary pressure is maintained. The actual simulation was performed for one half of the slab $0<x<1$, with the reflective boundary condition at $x=0$.

As is shown in Figs. 7 and 8 , at $t<1 / c_{0}=0.143007$ the head of the rarefaction wave $A$ propagates towards the slab center $x=0$ with the initial sound velocity $c_{0}=6.99269$ in the unperturbed matter. At point $B$ in Fig. 6, where the unloading isentrope crosses the binodal and the sound velocity experiences a jump from $c_{B+}=3.379472$ upstream to $c_{B-}=0.314276$ downstream, a region of constant flow (a binodal shelf) develops [1, 22]; in Figs. 7 and 8 the binodal shelf manifests itself as a plateau $B_{+} B_{-}$on the density profile. Note that, if we had $c_{B+}<c_{B-}$, the flow would develop a discontinuity, i.e. a rarefaction shock.

Formation of the binodal shelf is explained by the fact that with respect to any fixed "phase" of the rarefaction wave (i.e. with respect to any point with a given fixed thermodynamic state) the local flow velocity is equal to the local speed of sound. Therefore, because the inflow velocity $c_{B+}$ of the expanding material "into" the phase point $B$ is higher than the corresponding outflow velocity $c_{B-}$, material accumulates in the thermodynamic state $B$ as an ever expanding uniform layer; the width of this layer $\left|x_{B_{+}}-x_{B_{-}}\right|=\left(c_{B+}-c_{B-}\right) t$ grows linearly with time 


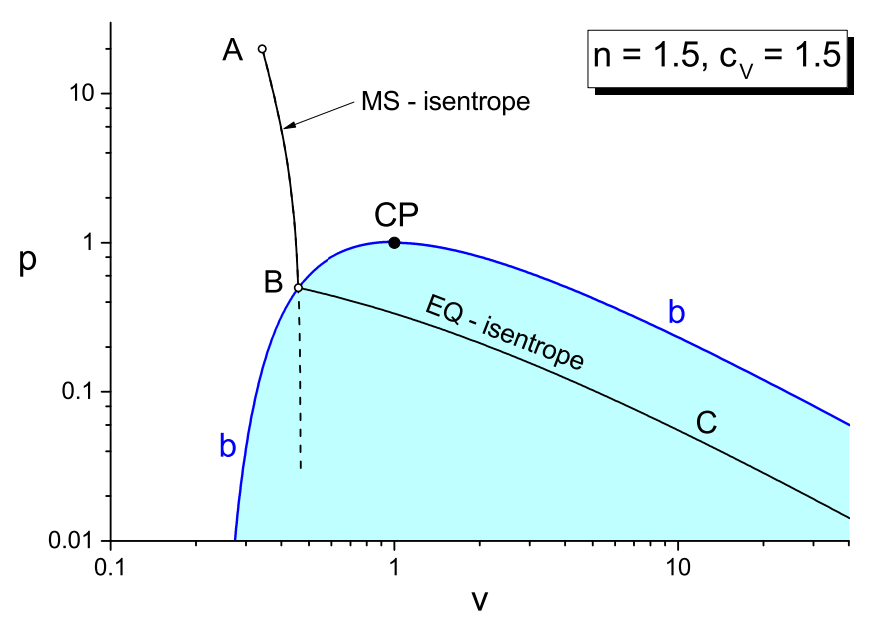

Figure 6: The unloading isentrope for $n=1.5, c_{V}=1.5$, starting with the initial state at point $A$ with $v=v_{0}=0.342238, p=p_{0}=19.9966$. It crosses the binodal $b$ at point $B$ with $v=v_{b x}=0.458694, p=p_{b x}=0.50002$. Note that the EQ-segment $C$ of the isentrope never comes out from the phase coexistence region under the binodal.

because for $t<1 / c_{0}$ the rarefaction flow is self-similar, where any fixed value of the similarity variable $\xi=x /\left(c_{0} t\right)$ corresponds to some fixed "phase" of the wave.

In Fig. 7 the numerical DEIRA solution (blue) is compared with the exact self-similar solution (red) for $t=0.1$. Apart from the corner points $A, B_{+}$and $B_{-}$, the numerical solution deviates from the exact one by no more than $\pm 0.2 \%$. The insert in this figure illustrates the smoothing of the numerical solution near the corner points by artificial viscosity in the DEIRA code.

After the head $A$ of the rarefaction wave reflects from the slab center at $t=$ $1 / c_{0}=0.143007$, there forms a central depression (a "hole") $D D$ on the density (pressure) profile, shown in Fig. 8 (magenta curve) for time $t=0.4$. As time goes on, the central depression becomes deeper, while the width of the binodal shelf decreases linearly in time with a speed $2 c_{B-}$. The insert in Fig. 8 with yellow background shows a blow-up of a narrow region around point $D$ on the density profile for $t=0.4$. It illustrates the quality of the DEIRA simulation with the in-line use of the EQ-GWEOS. One clearly sees that, in contrast to similar numerical results from Ref. [22], the numerical noise in our case is very low, namely, on the order of $\pm 10^{-4}$. The DEIRA simulation was performed on a grid of 2000 Lagrangian cells over the initial interval of $0<x<1$, of which 1500 cells had constant size within the $0<x<0.9$ interval whereas the 500 cells at $0.9<x<1.0$ had a progressively diminishing size (down to $\Delta x=3.56 \times 10^{-5}$ at $x=1$ ) towards the outer boundary. The simulation was done with only a linear component of the artificial viscosity [23] and the safety factor of 0.05 in the Courant-Friedrichs-Lewy limit for the time step; the simulation took about one 


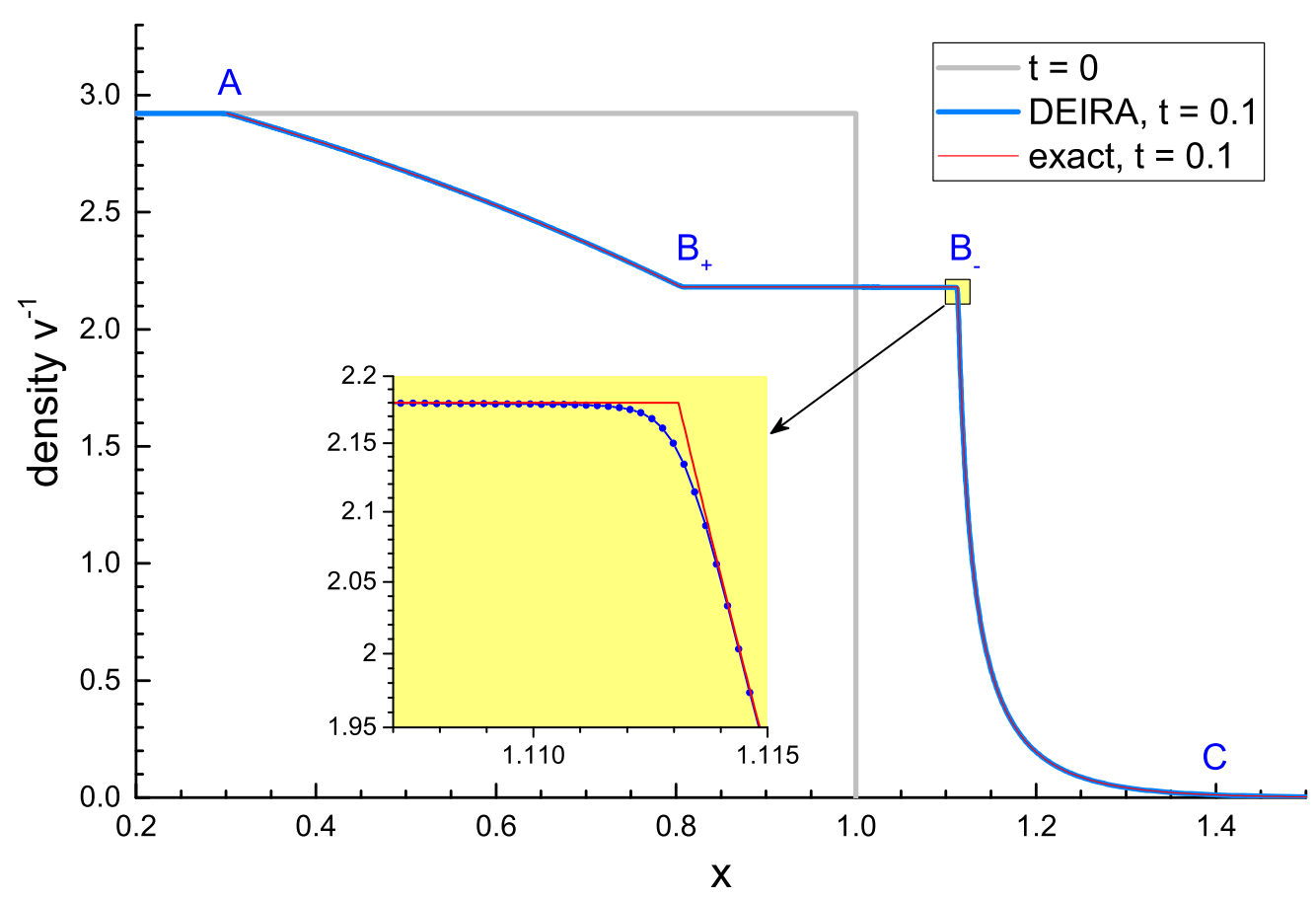

Figure 7: Density profile at $t=0.1$ in the rarefaction wave by unloading into vacuum along the isentrope shown in Fig. 6 for the EQ-GWEOS with $n=1.5$, $c_{V}=1.5$. The numerical solution (blue curve) is compared with the exact selfsimilar solution (red curve). The insert against the yellow background, where the individual grid-cell values are indicated as blue dots, illustrates numerical smoothing near the corner points, where the exact solution has discontinuous first derivative.

hour on a regular PC.

\section{Conclusion}

This work demonstrates that a relatively simple generalization of the van der Waals EOS, where the power exponent $n$ in the attractive term is treated as a free parameter, brings in several important advantages over the classical van der Waals formula. First of all, it allows to improve the agreement with the experimental data on the EOS of particular substances, either in terms of the critical compressibility factor $Z_{c r}$, or the ratio $\Lambda$ of the cohesive energy to the critical temperature. Second, variation of $n$ gives a possibility to distinguish between two qualitatively different situations for isentropic penetration into the region of metastable vapor: one, where any unloading isentrope inevitably enters the domain of absolute thermodynamic instability, and the other, where no penetration into the absolutely unstable domain occurs for sufficiently high isentropes. Third, it provides an additional lever for control over the region of 


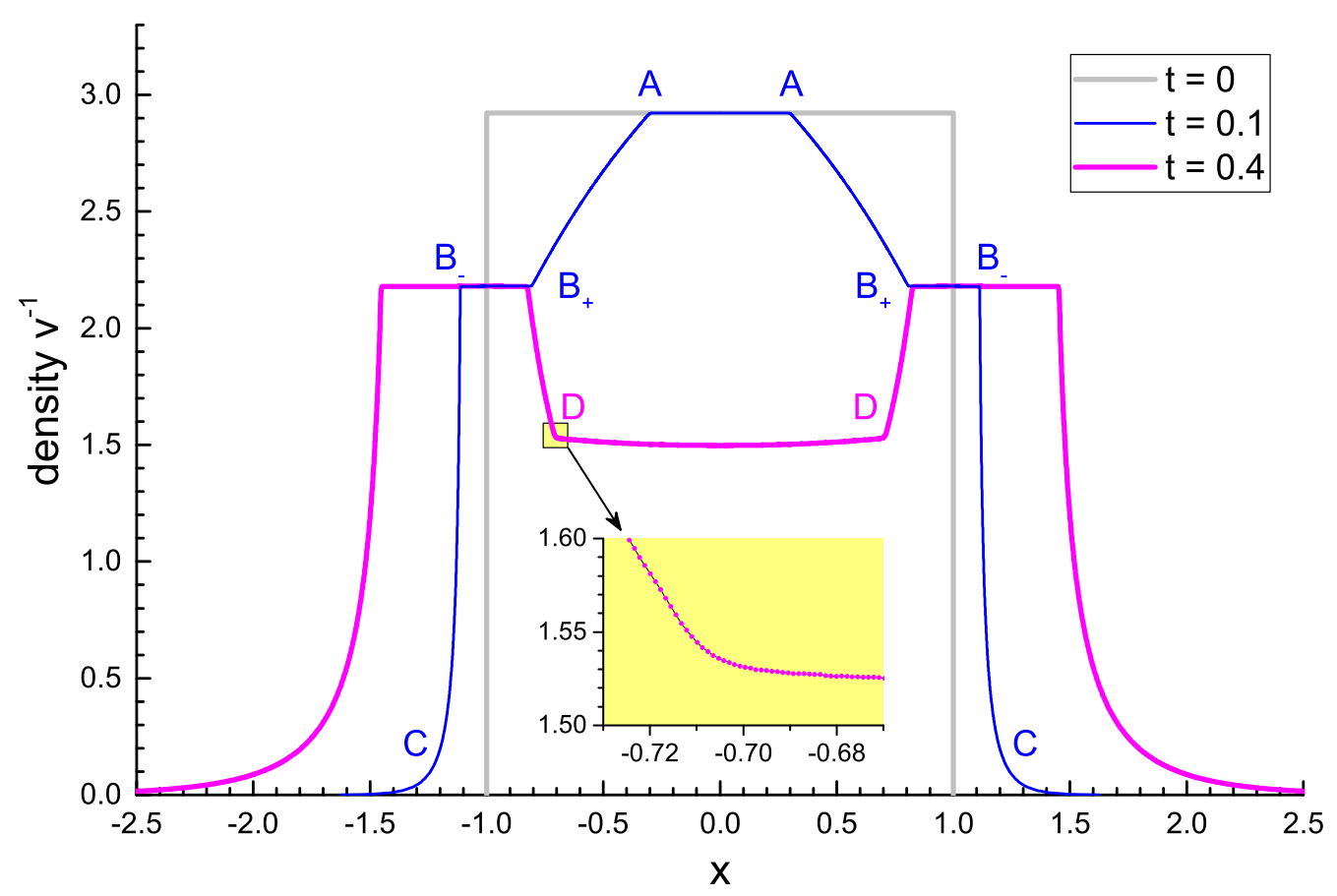

Figure 8: Density profiles at three characteristic times by unloading of an initially uniform planar slab into vacuum along the isentrope shown in Fig. 6 as calculated by the $1 \mathrm{D}$ DEIRA code for the EQ-GWEOS with $n=1.5, c_{V}=1.5$.

negative non-linearity.

At the same time, the present GWEOS model preserves a relative analytic simplicity of the original van der Waals formula. As a consequence, it provides an opportunity to incorporate its fully equilibrium branch (the MS-GWEOS) where the Maxwell construction must be invoked in the phase coexistence region - directly into 1D hydrodynamic codes for the in-line use. Numerical tests with the 1D Lagrangian DEIRA code have demonstrated that, despite the fact that the in-line Maxwell construction slows down EOS calculation by about a factor 100, it can still be afforded in 1D hydro codes without parallelization.

\section{References}

[1] K. Sokolowski-Tinten, J. Bialkowski, A. Cavalleri, D. von der Linde, A. Oparin, J. Meyer ter Vehn, and S. I. Anisimov. Transient states of matter during short pulse laser ablation. Phys. Rev. Lett., 81:224-227, Jul 1998.

[2] M. M. Basko, M. S. Krivokorytov, A. Yu. Vinokhodov, Yu. V. Sidelnikov, V. M. Krivtsun, V. V. Medvedev, D. A. Kim, V. O. Kompanets, A. A. Lash, and K. N. Koshelev. Fragmentation dynamics of liquid-metal droplets under ultra-short laser pulses. Laser Physics Letters, 14(3):036001, 2017. 
[3] S. Faik, M. M. Basko, An. Tauschwitz, I. Iosilevskiy, and J. A. Maruhn. Dynamics of volumetrically heated matter passing through the liquid-vapor metastable states. High Energy Density Physics, 8(4):349-359, 2012.

[4] L. D. Landau and E. M. Lifshitz. Statistical Physics. Butterworth Heinemann, 3 edition, 1996.

[5] W. G. Hoover, G. Stell, E. Goldmark, and G. D. Degani. Generalized van der Waals equation of state. The Journal of Chemical Physics, 63(12):5434-5438, 1975.

[6] D. Jou and C. Pérez-García. Generalized van der Waals equation for nonequilibrium fluids. Phys. Rev. A, 28:2541-2543, Oct 1983.

[7] M. M. Martynyuk and R. Balasubramanian. Equation of state for fluid alkali metals: Binodal. International Journal of Thermophysics, 16(2):533-543, Mar 1995.

[8] A. A. Sobko. Description of evaporation curve by the generalized vander-Waals-Berthelot equation. part i. Journal of Physical Science and Application), 4(8):524-530, 2014.

[9] R. Balasubramanian and K. Sugumar. A new three-parameter generalized van der Waals equation of state for water, heavy water, tritium oxide and silica. International Journal of Science and Research, 6(3):1130-1134, 2017.

[10] M. M. Martynyuk. Generalized van der Waals equation of state for liquids and gases. Zh. Fiz. Khim., 65:1716-1717, 1991.

[11] M. M. Martynyuk. Transition of liquid metals into vapor in the process of pulse heating by current. International Journal of Thermophysics, 14(3):457470, May 1993.

[12] M. M. Basko. DEIRA: A 1-D 3-T hydrodynamic code for simulating ICF targets driven by fast ion beams. Version 4, 2001; http://www.basko.net/mm/deira.

[13] Ya Song Wei and R. J. Sadus. Equations of state for the calculation of fluid-phase equilibria. AIChE Journal, 46(1):169-196, 2000.

[14] V. P. Skripov. Metastable Liquids. Wiley, New York, 1974.

[15] I. S. Grigoriev and E. Z. Meilikhov. Handbook of Physical Quantities. CRCPress, 1997. 
[16] P. A. Thompson. A fundamental derivative in gasdynamics. Physics of Fluids (1958-1988), 14(9):1843-1849, 1971.

[17] Ya. B. Zel'dovich. On the possibility of rarefaction shock waves. Zh. Eksp. Teor. Fiz., 4:363, 1946.

[18] M. S. Cramer and R. Sen. Exact solutions for sonic shocks in van der Waals gases. Physics of Fluids (1958-1988), 30(2):377-385, 1987.

[19] N. Zhao, A. Mentrelli, T. Ruggeri, and M. Sugiyama. Admissible shock waves and shock-induced phase transitions in a van der Waals fluid. Physics of Fluids, 23(8):086101, 2011.

[20] Ya. B. Zel'dovich and Yu. P. Raizer. Physics of Shock Waves and HighTemperature Hydrodynamic Phenomena. Dover Books on Physics. Dover Publications, 2012.

[21] M. N. Berberan-Santos, E. N. Bodunov, and L. Pogliani. The van der Waals equation: analytical and approximate solutions. Journal of Mathematical Chemistry, 43(4):1437-1457, May 2008.

[22] S. I. Anisimov, N. A. Inogamov, A. M. Oparin, B. Rethfeld, T. Yabe, M. Ogawa, and V. E. Fortov. Pulsed laser evaporation: equation-of-state effects. Applied Physics A, 69(6):617-620, Dec 1999.

[23] M. M. Basko. The method of artificial viscosity for computing onedimensional flows. USSR Computational Mathematics and Mathematical Physics, 30(2):176-182, 1990. 


\section{Contents}

1 Introduction 3

2 Parametrization of GWEOS 4

3 Properties of the MS-GWEOS $\quad 7$

3.1 The spinodal . . . . . . . . . . . . . . . . . . 8

3.2 Isentropes . . . . . . . . . . . . . . . . . . . . 10

3.3 Cohesive energy and realistic values of $n \ldots \ldots \ldots$

3.4 Negative non-linearity and rarefaction shocks . . . . . . . . . . 13

4 Fully equilibrium EQ-GWEOS $\quad 16$

4.1 The binodal . . . . . . . . . . . . . . . . . . . . 17

4.2 Thermodynamic functions of the EQ-GWEOS . . . . . . . . . 18

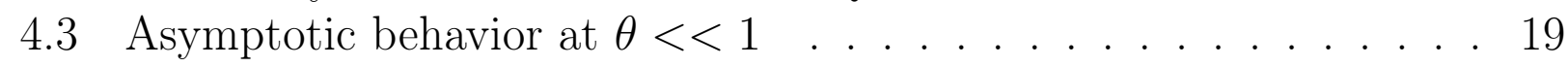

4.4 Expansion near the critical point . . . . . . . . . . . . 21

5 Illustrative example: unloading of a planar slab into vacuum 22

6 Conclusion $\quad 24$ 